\title{
Large-Eddy Simulation of Fuel Mixing Using Streamwise Vortices in a Supersonic Flow
}

\author{
By Junya WATANABE ${ }^{1)}$ Kenichi TAKITA $^{1)}$ and Tetsuji SUNAMI ${ }^{2}$ \\ ${ }^{1)}$ Department of Aerospace Engineering, Tohoku University, Sendai, Japan \\ ${ }^{2)}$ Kakuda Space Center, Japan Aerospace Exploration Agency, Miyagi, Japan
}

(Received June 16th, 2011)

\begin{abstract}
Turbulent mixing field of hydrogen jets injected in supersonic streamwise vortices was numerically investigated using large-eddy simulation (LES). The jet and the streamwise vortices were introduced in a similar and simplified manner to those introduced by the alternating-wedge (AW) strut injector. The LES reproduced the large-scale wavy jet structure containing small-scale vortices as those observed in the past experiment of the AW strut. The streamwise vortices strongly promoted the jet spreading and the turbulent transition. Downstream of the turbulent transition, the fully developed turbulent state was achieved in the wavy jet structure. The instantaneous and time-averaged mixing efficiencies were quantitatively evaluated and compared each other to investigate the intermittency of mixing state. The mixing efficiency rapidly increased by the turbulent transition. The jet mixing in the streamwise vortices had lower intermittency compared to the transverse jet from the wall. The effects of the incident shock wave across the hydrogen jet were also investigated in detail. The turbulent properties such as the fluctuation intensity and the power spectrum hardly changed by the crossing of oblique shock wave. The mixing efficiency slightly increased after the oblique shock wave passed through the hydrogen jet.
\end{abstract}

Key Words: $\quad$ LES, Turbulent Mixing, Supersonic Flow, Streamwise Vortices

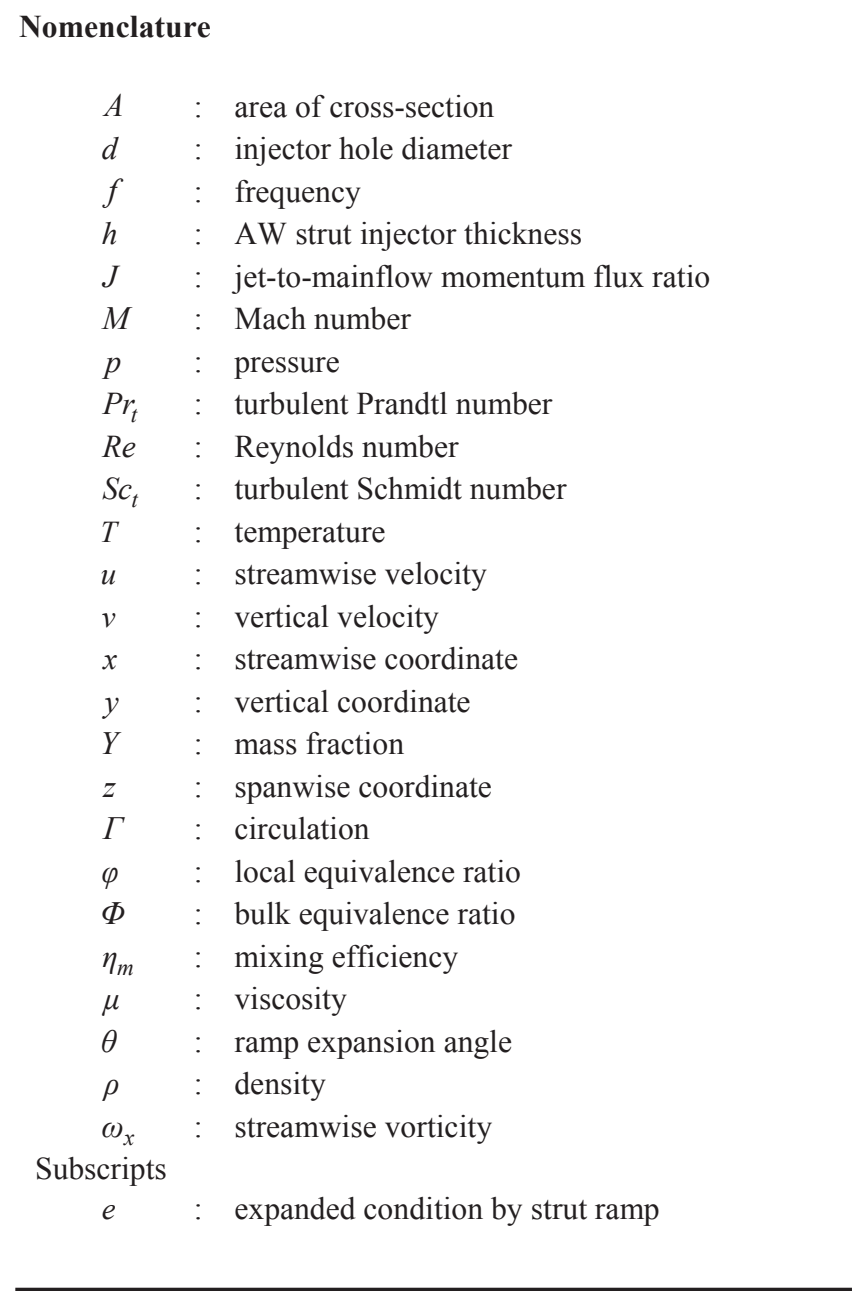

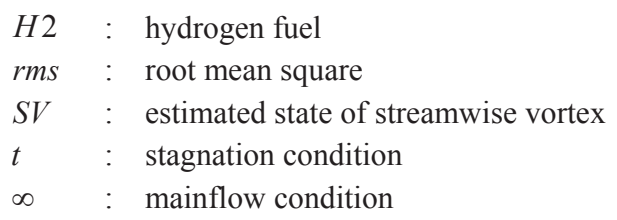

\section{Introduction}

Rapid mixing of fuel in a supersonic flow is one of the most important technical challenges for the success of supersonic combustor. The supersonic mixing is difficult due to the short residence time and the compressibility effects. As to the latter point, it is well known that the growth of lateral vortex is strongly suppressed under a high Mach number condition ${ }^{1,2)}$. Past numerical analysis ${ }^{3)}$ and experiment ${ }^{4)}$ revealed that the oblique vortex, which has the streamwise vorticity component, is prominent in a highly compressible mixing layer. This indicates that the use of streamwise vortices is effective for a supersonic mixing. Many researchers have developed fuel injector designs actively introducing streamwise vortices 5,6 . Sunami et al. ${ }^{6}$ proposed 'alternating-wedge (AW) strut injector'. Figure 1 shows the schematic of a typical AW strut injector. This strut has the characteristic rear-half geometry, namely the alternating upward and downward expansion ramps arranged in the spanwise direction. The spanwise row of counter-rotating streamwise vortices is introduced in the strut wake. The fuel injector holes locate between the upward and downward ramps. The fuel is injected parallel to the main airflow into each streamwise vortex core. Sunami et al. ${ }^{6}$ revealed by the combustion tests that the AW strut injector 
had superior mixing and combustion abilities to a generic strut injector without streamwise vortices. They also investigated the flow structure downstream of the AW strut using Mie scattering ${ }^{7)}$ and OH-PLIF ${ }^{8)}$. These visualizations showed instantaneous inner mixing and combustion fields by the streamwise vortices. However, the data is two-dimensional and does not give the quantitative mixing state, and the simultaneously obtainable data is limited. Therefore, the detailed understanding of flow structure and the quantitative mixing evaluation have not achieved yet. A numerical approach using a computational fluid dynamics (CFD) is effective for these purposes. Kodera et al. ${ }^{9)}$ conducted the CFD simulation of the AW strut injector. They showed the formation process of streamwise vortices interacting with the jet and their downstream development near the strut. However, their simulation did not reproduce small-scale turbulent eddies in the flowfield, which were observed in the experiments.

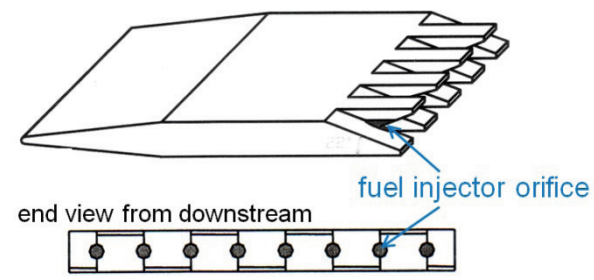

Fig. 1. Schematic of alternating-wedge (AW) strut injector.

In the present study, we investigate the turbulent mixing by the streamwise vortices in detail by simulating a simplified AW strut injector model. To reproduce the unsteady turbulent flow, large-eddy simulation (LES) is applied. In this work, the chemical reaction is not considered to focus on the turbulent mixing before ignition. The effects of streamwise vortex on turbulent mixing are revealed by simulating both cases with and without streamwise vortices. The spectrum analysis is also conducted to understand the turbulent state downstream. To investigate the state of streamwise vortex introduced by the AW strut, the circulation is assessed. Then, the mixing efficiency is evaluated. The effects of incident shock wave across the jet are also discussed.

\section{Numerical Methods}

\subsection{Numerical schemes}

The governing equations are the spatially filtered mass, momentum, total energy, and species conservation equations and the spatially filtered equation of state. For the subgrid scale (SGS) closure, the selective mixed-scale model ${ }^{10)}$ is used for the SGS stress. The SGS energy and species diffusions are modeled using an eddy-diffusivity assumption with $P r_{t}=0.9$ and $S c_{t}=0.9$. The SLAU ${ }^{11)}$ with the forth-order MUSCL approach ${ }^{12)}$ is used for the convective fluxes. The diffusion fluxes are computed using the second-order central differential scheme. The time advancement is performed with the third-order Runge-Kutta scheme. The present code is validated for compressible mixing layers ${ }^{13)}$ and a transverse jet injected into a supersonic crossflow ${ }^{14,15)}$. The detail of LES formula is described in Ref. 14.

\subsection{Computational grid}

Figure 2 illustrates the side view of computational grid. Grid generation and simulation for the exact geometry of the AW strut is difficult because of its complex geometry and would need the multi-block computation. To avoid these difficulties, only the downstream region of AW strut is simulated. The strut in this work has $h=5 \mathrm{~mm}$ and $\theta=14^{\circ}$. The computational domain is $24 h$ long, $4.8 h$ high and $4 h$ wide. The region framed by blue lines is the main domain where the grid spacing is constant in each direction. The grid has $321 \times$ $169 \times 141$ points in $x, y$ and $z$ directions in the main domain. The buffer region with the length of $8 h$ is located downstream of the main domain to damp a turbulent fluctuation. The flowfield in buffer region is out of discussion.

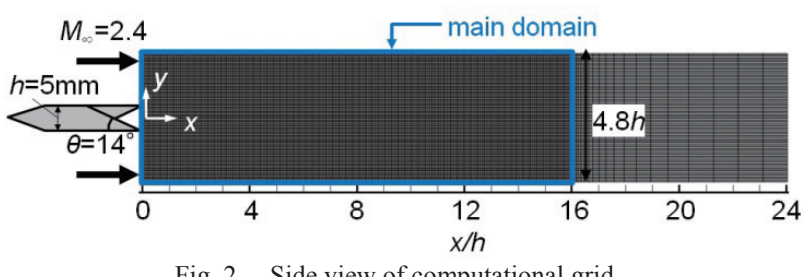

Fig. 2. Side view of computational grid.

\subsection{Boundary conditions}

The inflow condition is simply modeled in the inlet boundary to avoid the difficulties mentioned above. Figure 3 shows the simplified inflow setting at the inlet. The color map shows the Mach number distribution and the vectors show the in-plane velocity vectors. The flow expanded by the upward and downward ramps is simplified and modeled to be uniform and exist only in the strut region $(-0.5 \leq y / h \leq 0.5)$. The expanded flow condition in the strut region is calculated using the Prandtl-Meyer function. The mainflow conditions are $M_{\infty}$ $=2.4, p_{t \infty}=0.1 \mathrm{MPa}$, and $T_{t \infty}=290 \mathrm{~K}$. The mainflow Reynolds number based on the strut thickness is $\operatorname{Re}_{h}=5.3 \times 10^{4}$. The expanded conditions are $M_{e}=3.05$ and $v_{e}=149 \mathrm{~m} / \mathrm{s}$ in the case of $\theta=14^{\circ}$. The estimated circulation and Reynolds number of streamwise vortex are $\Gamma_{S V}=2 v_{e} h=1.49 \mathrm{~m}^{2} / \mathrm{s}$ and $R e_{S V}=\rho_{\infty} \Gamma_{S V} / \mu_{\infty}=2.7 \times 10^{4}$. Figure 4 shows the in-plane velocity vectors in the $x / h=4$ plane in the case without fuel jets. Four streamwise vortices are observed. We can confirm that the streamwise vortices are rapidly produced downstream by this simplified inflow condition. The present simplified inflow condition ignores the boundary layer on the strut surface. This may cause a difference from the experiment. However, by this simplification, we can observe the downstream development of a jet injected in a streamwise vortex without extra effects of the strut boundary layer.

The fuel injection holes with $d=2 \mathrm{~mm}$ locate between the upward and downward expanded flows as shown in Fig. 3. The hydrogen fuel is injected parallel to the mainflow at the sonic speed. The injection conditions are $T_{t H 2}=290 \mathrm{~K}, \Phi=$ 0.92 , and $J=1.91$. The injection condition is uniform and fixed throughout the calculation.

At the exit boundary, the variables are extrapolated from the inner point. The periodic condition is applied at the side $(z)$ boundaries. Either a slip wall or a free outflow condition is applied at the top and bottom boundaries. The shock wave 
reflects in a slip wall condition, not in a free outflow condition. By comparing the flow results in these two conditions, we can see the effect of incident shock wave across the hydrogen jet. The slip wall condition is basically applied unless we explicitly specify the condition.

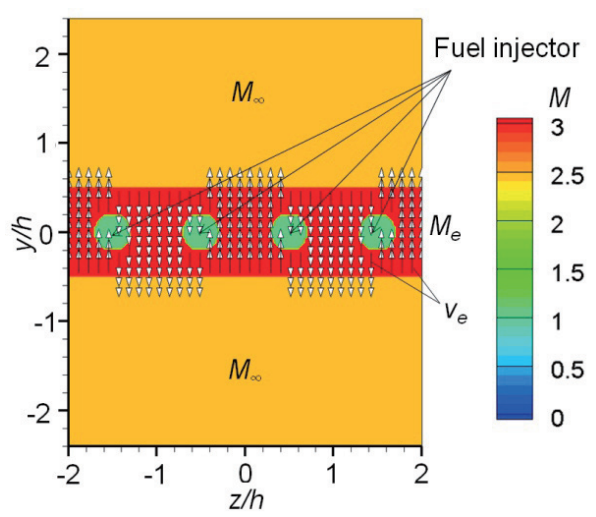

Fig. 3. Simplified inflow condition.

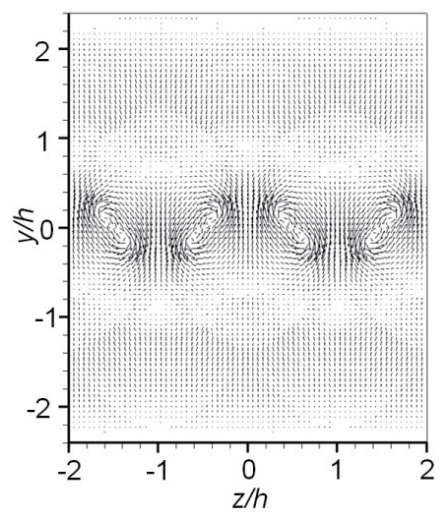

Fig. 4. In-plane velocity vectors in $x / h=4$ plane.

\section{Results and Discussion}

\subsection{Instantaneous flow structure}

Figure 5 shows instantaneous schlieren images produced from the LES results. The density gradient in $x$ direction is integrated in $z$ direction to obtain the $x-y$ schlieren image. Four cases are simulated: (a) only streamwise vortices (without fuel jets), (b) jets without streamwise vortices (without expanded state in the mainflow), (c) jets with streamwise vortices in the free outflow condition, and (d) jets with streamwise vortices in the slip wall condition. In the case (a), the turbulent structure cannot be observed and the flow is steady. In the case (b), the turbulence appears just near the exit of domain. In the cases (c) and (d), the turbulent structure prominently appears around $x / h=7$. By comparing these images between the cases (b) and (d), the jet spreading in the case (d) is much larger than that in the case (b). This is due to the vortex stretching by the induced velocity field of the counter-rotating streamwise vortices. It should be noted that the position of turbulent transition is much more upstream in the case (d). The streamwise vortices strongly enhance the instability to lead to the early turbulent transition. Then, the effect of incident shock wave is investigated by comparing the schlieren images for the cases (c) and (d). We can confirm that the incident shock wave does not exist in the case (c) although the weak expansion waves exist. The incident shock wave observed in the case (d) is steady without any fluctuation. Both the jet spreading and the position of turbulent transition are almost the same in the both cases. This indicates that the calm incident shock wave to the hydrogen jet does not primarily affect the turbulent transition of jet in the present case. The turbulent transition probably occurs due to the instability of shear layer between the hydrogen jet and the swirling mainflow. The combination use of the streamwise vortices and the jet which is injected into the streamwise vortices is much more important than the effect of steady incident shock wave for the turbulent transition.

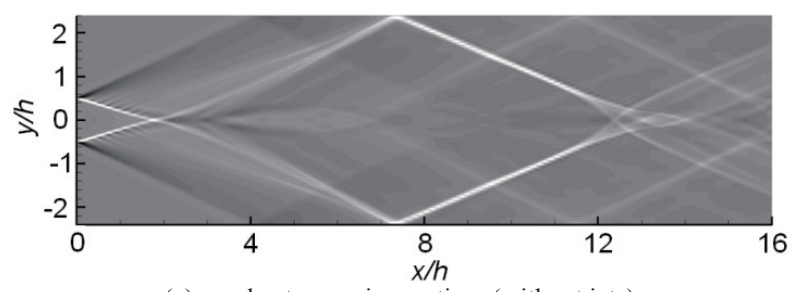

(a) only streamwise vortices (without jets)

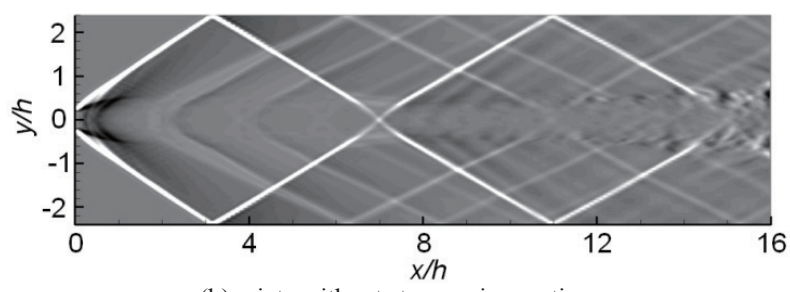

(b) jets without streamwise vortices

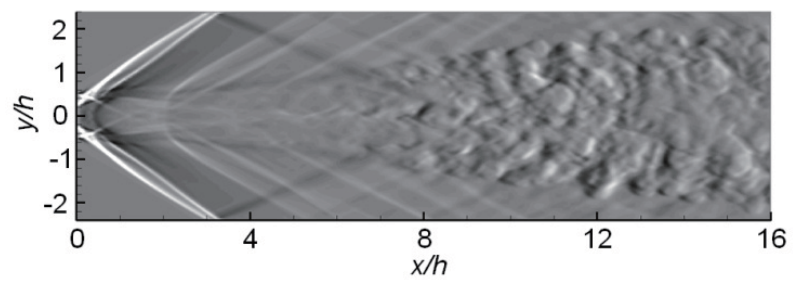

(c) jets with streamwise vortices (free outflow condition)

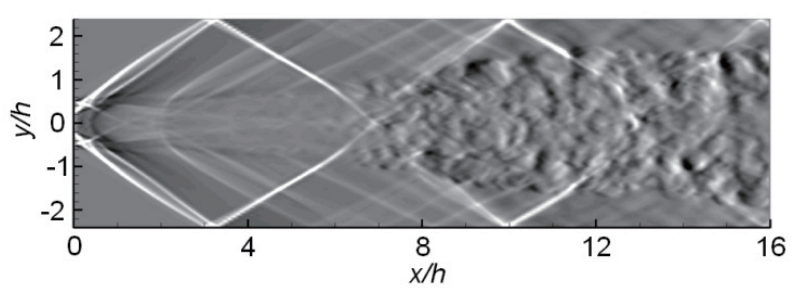

(d) jets with streamwise vortices (slip wall condition)

Fig. 5. Instantaneous pseudo schlieren images.

The inner structure of flowfield is discussed below for the jets with streamwise vortices in the slip wall condition. Figure 6 shows an instantaneous distribution of the hydrogen mass fraction in $x / h=0,2,4,6,8,10,12,14$ and 16 planes. The circular jet is initially entrained into the vortical region to form larger-scale circular swirling jet, which is almost identical to the streamwise vortex region. Subsequently, the jet is stretched toward the oblique direction in $y$-z plane due to the induced velocity field by the row of counter-rotating streamwise vortices. Then, the jet unites with the neighboring 
jets, and the large-scale wavy jet structure is formed downstream of $x / h=8$. In this wavy jet structure, a lot of small-scale structure generated. Similar large-scale wavy jet structures filled with the smaller-scale turbulent eddies were also observed in the past Mie scattering images ${ }^{7)}$.

Figure 7 shows the instantaneous streamwise vorticity distribution in the same planes and at the same instant as Fig. 6 . The magnitude of the streamwise vorticity rapidly increases at the position of turbulent transition. A lot of small-scale vortices crowd in the large-scale wavy structure. Further downstream, the magnitude of the streamwise vorticity gradually decreases and the vortical region diffuses to spread into wider area in the $y-z$ cross-section.

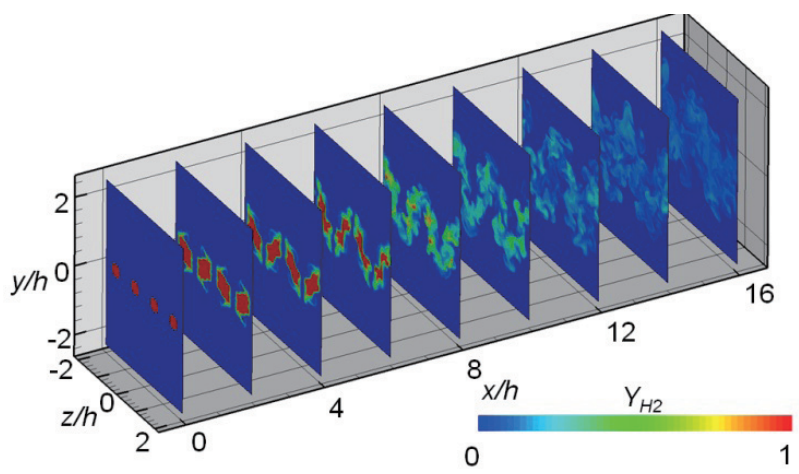

Fig. 6. Instantaneous hydrogen mass fraction distribution.

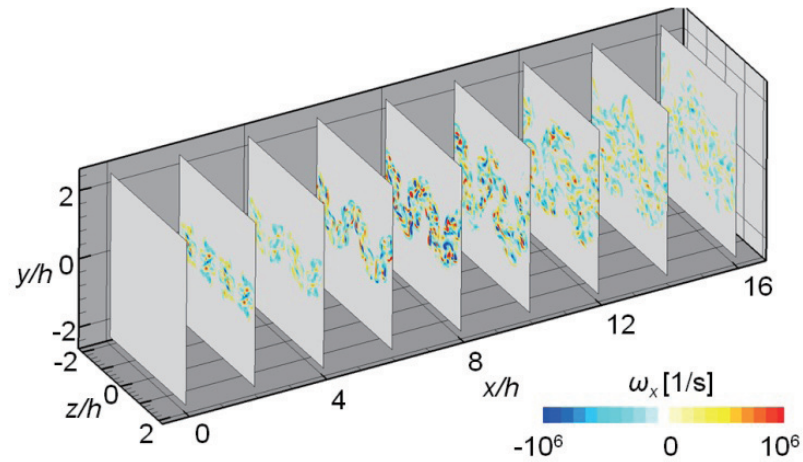

Fig. 7. Instantaneous streamwise vorticity distribution.

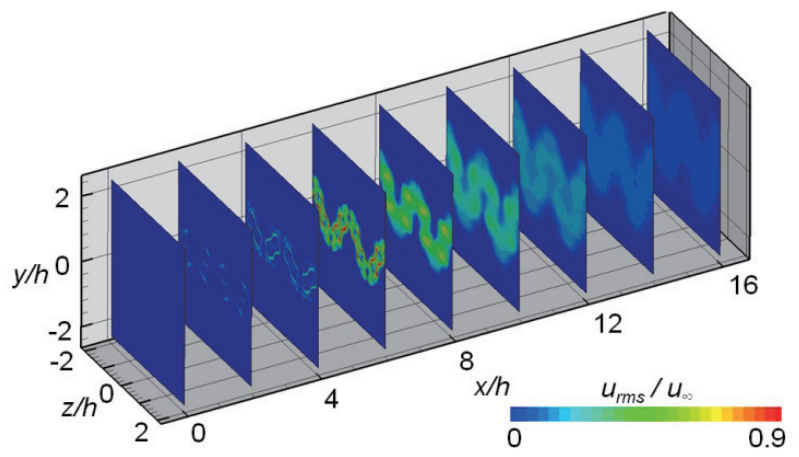

Fig. 8. RMS distribution of streamwise velocity fluctuation.

\subsection{Turbulent statistics}

Figure 8 shows the root mean square (RMS) distribution of streamwise velocity fluctuation in the same planes as Fig. 6. The RMS value of $u$ is normalized by $u_{\infty}$. The velocity fluctuation starts to increase in the shear layer between the jet and the mainflow. Downstream of the turbulent transition, the fluctuating region spreads and uniformly exists in the large-scale wavy structure. As going further downstream, the velocity fluctuation gradually decreases. The RMS distribution in the case of the free outflow condition is almost the same although we do not show here. This means that the steady incident shock wave does not drastically affect the magnitude of turbulent fluctuations.

Figure 9 shows the power spectra of streamwise velocity fluctuation at the center of the streamwise vortex $((y / h, z / h)=$ $(0,0.5))$ and between the neighboring streamwise vortices $((y / h, z / h)=(0,0))$ at two streamwise stations $(x / h=8$ and 16). The abscissa is the frequency normalized by $d$ and $u_{H 2}-u_{\infty}$. A line with $-5 / 3$ slope is also shown in the figure. At $x / h=8$, some prominent frequencies exist $\left(f d /\left(u_{H 2}-u_{\infty}\right) \sim 0.15,0.3\right.$, etc. $)$. The value of $f d /\left(u_{H 2}-u_{\infty}\right)=0.3$ is close to the frequency of preferred mode appearing in the free jet ${ }^{16)}$. The value of $f d /\left(u_{H 2}-u_{\infty}\right)=0.15$, which is about half of the larger prominent frequency, is considered to represent the vortex merging. The prominent frequencies at $(y / h, z / h)=(0,0.5)$ and $(0,0)$ are almost the same. The power spectrum at $(y / h, z / h)=(0,0.5)$ is larger than that at $(y / h, z / h)=(0,0)$ through the entire range of frequency, that is, the fluctuation at the center of streamwise vortex is larger than that between the streamwise vortices. Further downstream, at $x / h=16$, a prominent frequency disappears and the power spectrum is almost the same level each other. The inertial subrange where the spectra follows the $-5 / 3$ power low can be observed. This implies that the turbulence reaches fully developed state downstream. Also, we can confirm that the present LES resolves a wide range of scale including the inertial subrange.

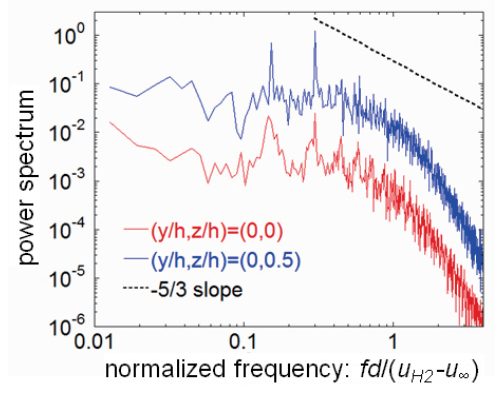

(a) $x / h=8$

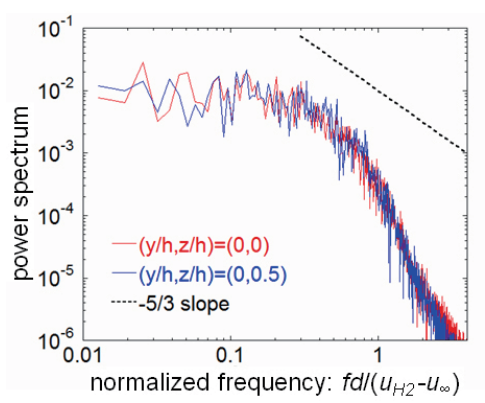

(b) $x / h=16$

Fig. 9. Power spectra of streamwise velocity fluctuation.

Figure 10 shows the comparison of power spectrum obtained in the slip wall and free outflow conditions. The power spectrum of streamwise velocity fluctuation at $(x / h, y / h$, 
$z / h)=(8,0,0)$ was compared. The magnitude and the prominent frequency are almost the same each other. This indicates that the steady incident shock wave does not drastically change the turbulent properties of jet.

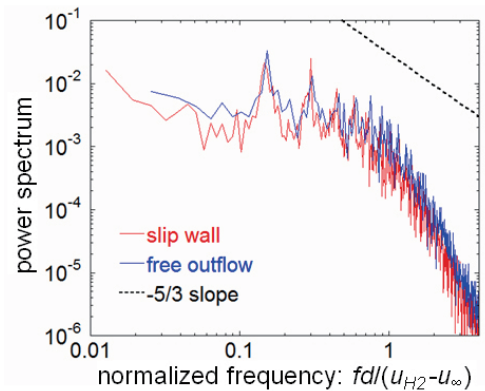

Fig. 10. Effect of incident shock wave on power spectrum.

\subsection{Evaluation of streamwise vortex}

To investigate the downstream development of streamwise vortex introduced, we evaluate the circulation by integrating the streamwise vorticity over the cross-section in the range from $z / h=0$ to $z / h=1$. Figure 11 shows the streamwise change of time-averaged circulation. Both the results in the slip wall and free outflow conditions are plotted in the figure. In the free outflow condition, where the effects of incident shock wave does not exist, the circulation rapidly increases around the turbulent transition and gradually decreases downstream of $x / h=8$. This decrease is considered to be caused by the vortex dissipation. Then, the effect of incident shock wave on the circulation is investigated. Large differences exist around the position where the reflected shock wave passes through the hydrogen jet. In the position of first incident shock wave, the rapid increase by the turbulent transition was delayed, but the maximum circulation is larger than that without the incident shock wave. In the position of second incident shock wave, the circulation rapidly decreases first, then increases again to the same level of that without the incident shock wave.

Next, we investigate the cause of circulation change observed in Fig. 11 using the vorticity equation. The equation of streamwise vorticity is expressed as

$$
\begin{array}{r}
\frac{D \omega_{x}}{D t}=-\omega_{x}\left(\frac{\partial v}{\partial y}+\frac{\partial w}{\partial z}\right)+\left(\omega_{y} \frac{\partial u}{\partial y}+\omega_{z} \frac{\partial u}{\partial z}\right) \\
+\frac{1}{\rho^{2}}\left(\frac{\partial \rho}{\partial y} \frac{\partial p}{\partial z}-\frac{\partial \rho}{\partial z} \frac{\partial p}{\partial y}\right)+(\text { viscos })
\end{array}
$$

The terms on the right hand side are the factors in the change of streamwise vorticity. The first term is the vorticity change by the dilatation and contraction of vortex. The second term is the vorticity change by the rotation of lateral vortex due to the presence of velocity gradients. The third term is the vorticity generation by the baroclinic torque. The forth term is the vorticity dissipation due to viscosity. Here, we compare the integrated values of the first, second and third terms over the cross-section to evaluate the contribution of each term for the circulation change. Figure 12 shows the comparison of each term calculated from the result in the slip wall condition at some streamwise stations. Around the position of turbulent transition, the rotation term strongly contributes to the circulation increase. That is, the streamwise vortex is strengthened by the rotation of lateral vortex at the turbulent transition position. At $x / h=12$ and 13 , where the second incident shock wave exists, the baroclinic torque term is dominant and corresponds to the circulation change in Fig. 11. The incident shock wave affects the state of streamwise vortex through the baloclinic torque for the hydrogen jets.

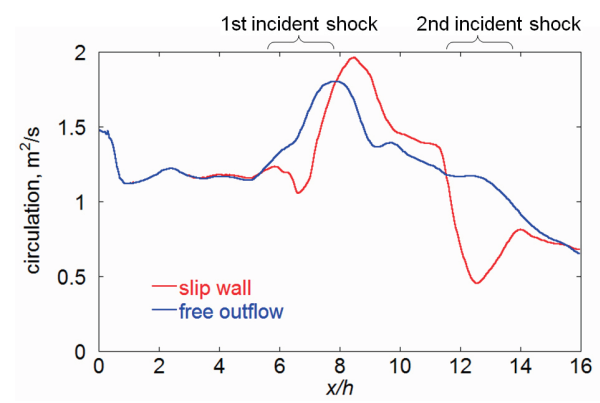

Fig. 11. Streamwise distribution of circulation.

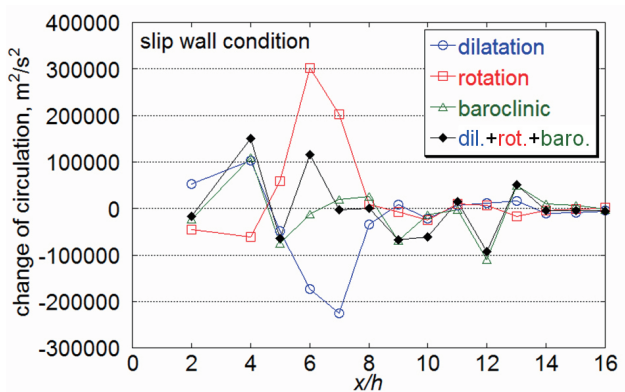

Fig. 12. Comparison of terms contributing to circulation change.

\subsection{Mixing efficiency}

In this section, we evaluate the mixing efficiency defined as the following equation.

$$
\eta_{m}=\frac{\int_{A}\left(\rho u Y_{H 2} / \varphi^{\prime}\right) d A}{\int_{A} \rho u Y_{H 2} d A} \quad \varphi^{\prime}= \begin{cases}1 & (\varphi \leq 1) \\ \varphi & (\varphi>1)\end{cases}
$$

The mixing efficiency should be evaluated using the instantaneous quantities, but a lot of conventional approach such as probe measurements and CFDs using Reynolds averaged equations (RANS) can evaluate only the mixing efficiency obtained from the averaged quantities. Here, we obtain the mixing efficiencies calculated from both instantaneous and averaged quantities using the LES results and compare their difference. The difference is owing to the intermittency of turbulent mixing flowfield.

Figure 13 shows the streamwise variation of mixing efficiency. The open symbols mean the average of 200 instantaneous mixing efficiencies sampled at intervals of $5 \mu \mathrm{s}$ and the error bars mean the standard deviations of the mixing efficiency fluctuation. The solid line shows the distribution of mixing efficiency calculated from the time-averaged quantities. The results in the free outflow and slip wall conditions are shown by blue and red, respectively. The case without streamwise vortices is also shown by green. The mixing efficiency in the cases with streamwise vortices is much larger than that in the case without streamwise vortices. The streamwise vortices strongly enhance the fuel mixing. In 
the cases with streamwise vortices, the mixing efficiency rapidly increases around $x / h=7$, where the turbulent transition occurs, and almost linearly increases further downstream. By comparing the difference between the time-averaged and instantaneous mixing efficiencies, the time-averaged one is larger than the instantaneous one by $15-20 \%$. This difference is relatively small compared to the case of transverse jet injected from the wall into a supersonic crossflow, where our past LES ${ }^{15)}$ showed the time-averaged mixing efficiency to be about twice as large as the instantaneous one. Therefore, the fuel injection into the streamwise vortices by the AW strut injector achieves relatively stable and uniform mixing with lower intermittency compared to the wall transverse injection.

Then, the effect of incident shock wave on the mixing is discussed by comparison between the slip wall and free outflow conditions. The mixing efficiency somewhat increases after the reflected shock wave passes through the hydrogen jet (around $x / h=8$ and 14). The increase in mixing efficiency is just 0.02 by the present steady incident shock wave. The effect of the incident shock wave on the mixing is not large. We should note that if the stronger or fluctuating shock wave passes through the hydrogen jet, the increase in mixing efficiency could be more drastic.

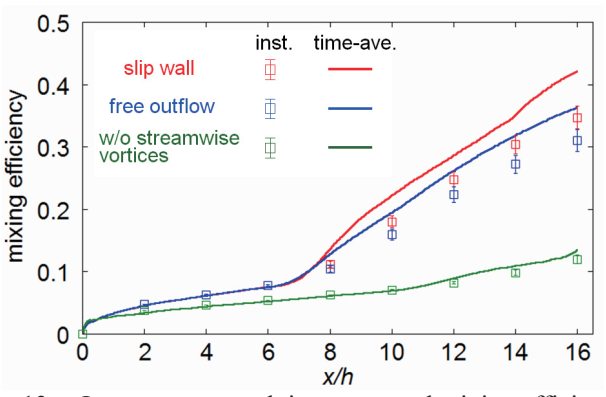

Fig. 13. Instantaneous and time-averaged mixing efficiencies.

\section{Conclusions}

In this study, the turbulent flowfield of parallel hydrogen jets injected into supersonic streamwise vortices were simulated using LES. The followings are the conclusions obtained from the present study.

- The large-scale wavy jet structure containing a lot of small-scale vortices was formed downstream. Prominent frequencies of turbulence exist near the turbulent transition, but not downstream enough. The fully developed turbulent state probably obtained in the large-scale wavy structure.

- The introduction of streamwise vortices strongly enhanced the instability of shear layer between the hydrogen jet and the main airflow to achieve turbulent mixing much more upstream.

- The mixing efficiency rapidly increased by the turbulent transition and almost linearly increased downstream.

- The mixing state in the AW strut injector had lower intermittency compared to that in the transverse wall injection. - The steady incident shock wave across the hydrogen jet changed the circulation of streamwise vortex through the baroclinic torque, but hardly changed the turbulent intensity and spectrum property. The mixing efficiency slightly increased after the oblique shock wave passed through the hydrogen jet.

\section{Acknowledgments}

This work was supported by Grant-in-Aid for Scientific Research (S), 21226020, and for JSPS Fellows, 21.4309. The computation was conducted using supercomputing resources at the Cyberscience Center, Tohoku University.

\section{References}

1) Bodanoff, D. W.: Compressibility Effects in Turbulent Shear Layer, AIAA J., 21 (1983), pp. 926-927.

2) Papamoschou, D. and Roshko, A.: The Compressible Turbulent Shear Layer: An Experimental Study, J. Fluid Mech., 197 (1988), pp.453-477.

3) Sandham, N. D. and Reynolds, W. C.: Three-Dimensional Simulations of Large Eddies in the Compressible Mixing Layer, $J$. Fluid Mech., 224 (1991), pp. 133-158.

4) Clemens, N. T. and Mungal, M. G.: Large-Scale Structure and Entrainment in the Supersonic Mixing Layer, J. Fluid Mech., 284 (1995), pp. 171-216.

5) Northam, G. B., Greenberg, I. and Byington, C. S.: Evaluation of Parallel Injector Configurations for Supersonic Combustion, AIAA Paper 89-2525 (1989).

6) Sunami, T., Wendt, M. N. and Nishioka, M.: Supersonic Mixing and Combustion Control Using Streamwise Vortices, AIAA Paper 98-3271 (1998).

7) Sunami, T. and Scheel, F.: Analysis of Mixing Enhancement Using Streamwise Vortices in a Supersonic Combustor by Application of Laser Diagnostics, AIAA Paper 2002-5203 (2002).

8) Sunami, T., Magre, P., Bresson, A., Grisch, F., Orain, M. and Kodera, M.: Experimental Study of Strut Injectors in a Supersonic Combustor Using OH-PLIF, AIAA Paper 2005-3304 (2005).

9) Kodera, M., Sunami, T. and Scheel, F.: Numerical Study on the Supersonic Mixing Enhancement Using Streamwise Vortices, AIAA Paper 2002-5117 (2002).

10) Lenormand, E., Sagaut, P. and Ta Phuoc, L.: Large Eddy Simulation of Subsonic and Supersonic Channel Flow at Moderate Reynolds Number, International Journal for Numerical Methods in Fluids, 32 (2000), pp. 369-406.

11) Shima, E. and Kitamura, K.: On New Simple Low-Dissipation Scheme of AUSM-Family for All Speeds, AIAA Paper 2009-136 (2009).

12) Yamamoto, S. and Daiguji, H.: Higher-Order-Accurate Upwind Schemes for Solving the Compressible Euler and Navier-Stokes Equations, Computers and Fluids, 22 (1993), pp. 259-270.

13) Watanabe, J., Kouchi, T., Takita, K. and Masuya, G.: Large-Eddy Simulation of Compressible Mixing Layers, Proceedings of the 7th International Conference on Flow Dynamics, 2010, pp. 540-541.

14) Watanabe, J., Kouchi, T., Takita, K. and Masuya, G.: Numerical Study on Turbulent Structure of Transverse Jet into a Supersonic Flow, AIAA J. (to be published).

15) Watanabe, J., Kouchi, T., Takita, K. and Masuya, G.: Large-Eddy Simulation of Turbulent Mixing Mechanism of Hydrogen and Ethylene fuels in Supersonic Combustor, Proceedings of the 48th Symposium (Japanese) on Combustion, 2010, pp. 98-99, (in Japanese).

16) Petersen, R. A. and Samet, M. M.: On the Preferred Mode of Jet Instability, J. Fluid Mech., 194 (1988), pp. 153-157. 